\title{
Efecto de Tetraselmis chuii, Nannochloris oculata y Dunaliella salina sobre el crecimiento poblacional de Apocyclops distans (Copepoda,Cyclopoidae) en diferentes condiciones de temperatura e iluminación
}

\author{
Effect of Tetraselmis chuii, Nannochloris oculata and Dunaliella salina \\ on the population growth of Apocyclops distans (Copepoda, Cyclopoidae) \\ at different temperature and ligth conditions
}

\author{
Aidé Velásquez ${ }^{1}$, Jesús Rosas ${ }^{2}$, Tomas Cabrera ${ }^{1}$, José Millán ${ }^{2}$ y Miguel Hernández ${ }^{1}$ \\ ${ }^{1}$ Escuela de Ciencias Aplicadas del Mar \\ ${ }^{2}$ Instituto de Investigaciones Científicas \\ Universidad de Oriente, Isla de Margarita. Venezuela. \\ rosas@ne.udo.edu.ve
}

\begin{abstract}
Resumen.- En el presente trabajo se estudia el efecto de Tetraselmis chuii, Nannochloris oculata y Dunaliella salina en el crecimiento poblacional de Apocyclops distans (Kiefer, 1956) cultivado bajo diferentes condiciones de luz y temperatura. Durante 10 días se cultivó $A$. distans ( $5 \mathrm{ind} / \mathrm{mL}$ ) en tubos de ensayos (540) conteniendo $5 \mathrm{~mL}$ de agua de mar inoculada con las microalgas antes mencionadas como dietas unialgales, a temperatura ambiente $\left(26-33,8^{\circ} \mathrm{C}\right)$ y en el laboratorio $\left(22 \pm 1^{\circ} \mathrm{C}\right)$ a tres condiciones de iluminación, fotoperiodo (12 h luz: $12 \mathrm{~h}$ oscuridad), oscuridad y luz continua; cada tratamiento se realizó por triplicado. La mayor producción de nauplios (105,33 \pm $48,58 / 5 \mathrm{~mL}$ ) se obtuvo con $T$. chuii, en oscuridad a temperatura ambiente $\left(26-33,8{ }^{\circ} \mathrm{C}\right)$ el tercer día de cultivo. Los análisis estadísticos señalan que el factor que influyó en la producción de nauplios fue la temperatura $(\mathrm{P} \leq 0,05)$. La mayor producción de copepoditos fue de $25,33 \pm 11,93 / 5 \mathrm{~mL}$ con $T$. chuii, bajo fotoperíodo (12h luz: $12 \mathrm{~h}$ oscuridad) y temperatura ambiente $(26-33,8)$ el sexto día. Los análisis estadísticos indican que el fotoperíodo y la dieta a base de $T$. chuii y $N$. oculata produjeron diferencias significativas en el número de copepoditos. El mayor número promedio de hembras adultas no ovadas $(26,66 \pm$ $36,66 / 5 \mathrm{~mL}$ ) con $T$. chuii se produjo el sexto día a temperatura ambiente. El promedio máximo de hembras adultas ovadas $(9,66$ $\pm 5,50 / 5 \mathrm{~mL}$ ) con $T$. chuii se produjo el tercer día a temperatura ambiente en oscuridad. El mayor número de masas ovígeras desprendidas fue de $27,33 \pm 17,92 / 5 \mathrm{~mL}$ con $N$. oculata a temperatura ambiente. El mayor número promedio del total de copépodos $(132,66 \pm 34,00 / 5 \mathrm{~mL})$ con $T$. chuii se obtuvo en oscuridad a temperatura ambiente el tercer día. Los resultados alcanzados con $D$. salina, no produjeron diferencias en el crecimiento poblacional de $A$. distans $(\mathrm{P} \leq 0,05)$. Se concluye que A. distans logro desarrollarse completamente y presentó una evidente preferencia por la especie $T$. chuii, microalga de mayor volumen celular y movilidad entre las tres utilizadas. Las microalgas $N$. oculata y $D$. salina produjeron valores promedio bajos en el número total de copépodos, aunque permitieron el desarrollo completo de A. distans.

Palabras clave: Copépodos, alimentación, microalgas, Apocyclops distans.
\end{abstract}

\begin{abstract}
Several experiments were conduced to determine the effect of the microalgae Tetraselmis chuii, Nannochloris oculata and Dunaliella salina on the population growth of Apocyclops distans (Kiefer, 1956) at different temperature and light conditions. During 10 days A. distans (5 ind $/ \mathrm{mL}$ ) was cultured in tested tubes (540) containing $5 \mathrm{~mL}$ of seawater inoculated with the microalgae last mencionated as unialgal diets, at natural temperature $\left(26-33,8{ }^{\circ} \mathrm{C}\right)$ and lab temperature $(22 \pm$ $1^{\circ} \mathrm{C}$ ) under three light conditions, photoperiod (12 h light: $12 \mathrm{~h}$ dark), dark and continuous light; each was triplicated. The maximum value of nauplii $(105.33 \pm 48.58 / 5 \mathrm{~mL})$ and copepodits production $(25.33 \pm 11.93 / 5 \mathrm{~mL})$ was obtained in dark and natural temperature at the third day and natural photoperiod and temperature to sixth culture day respectively using $T$. chuii. The statistical analysis showed that temperature was the most important factor in the nauplii production with $T$. chuii and photoperiod and diet on the copepodits production. The highest average value of adult females carrying no eggs (26.66 \pm $36.66 / 5 \mathrm{~mL})$ and adult females carrying eggs $(9.66 \pm 5.50 / 5 \mathrm{~mL})$ was attained at the sixth day under natural temperature and third culture day under natural temperature and dark respectively, with T. chuii. The maximum average value of liberated eggs mass $(27.33 \pm 17.92 / 5 \mathrm{~mL})$ was produced at natural temperature with $N$. oculata, the light was not significant $(\mathrm{P} \leq 0.01)$. The highest total number of copepods attained was $132.66 \pm 34.00 / 5 \mathrm{~mL}$ under dark and natural temperature at the third day with $T$. chuii. The results obtained with $D$. salina were not significant on the population growth of $A$. distans ( $\mathrm{P} \leq 0.05)$. It was concluded that A. distans obtained a complete development and to prefer $T$. chuii, the highest volume and mobility of three microalgae used $N$. oculata and D. salina microalgae produced the lowest total copepods number average values, although they permitted the complete development of $A$. distans.
\end{abstract}

Key words: Copepods, feeding, microalgae, Apocyclops distans.

\section{Introducción}

En acuicultura, los estudios han demostrado que los copépodos tienen mayor valor nutritivo que Artemia, porque cubren mejor los requerimientos nutritivos de larvas de peces marinos, además, pueden ser administrados como nauplios, copepoditos o adultos. Los copépodos poseen un contenido de proteína de 44 a 52\% con un buen perfil de aminoácidos, a excepción de 
metionina e histidina. Otra ventaja de los copépodos es el contenido de ácidos grasos insaturados (HUFA) así como la calidad y cantidad de contenido de enzimas digestivas presentes en los copepoditos y adultos, que juegan un rol importantísimo como alimento de las primeras fases larvales de peces y crustáceos (Lavens \& Sorgeloos 1996, Heath \& Moore 1997, Stottrup \& Norsker 1997). Su típico movimiento en zig-zag, es un importante estímulo de aceptabilidad para muchos peces, los cuales los prefieren antes que a los rotíferos, otra ventaja del uso de los copépodos es que las especies bentónicas como Tisbe, mantienen las paredes y el fondo de los estanques de cultivo libres de microalgas y detritos por el pastoreo (Lavens \& Sorgeloos 1996).

Varias especies de copépodos han sido ampliamente estudiados para su producción en masa entre las que destaca Apocyclops royi (Su et al. 1997). El género Apocyclops (=Metacyclops) es de amplia distribución en regiones tropicales y templadas, siendo más prolífero en Europa, Africa y Sur América. La especie Apocyclops distans (= Metacyclops distans) fue reportada por primera vez para Venezuela en el lago de Maracaibo por Kiefer (1956), es típica habitante de lagunas costeras y estuarios, en un amplio rango de salinidad y otros factores ambientales (Rodríguez 1999).

En Venezuela se ha estudiado la importancia de copépodos en acuicultura (Rosas et al. 1993), el cultivo masivo y la composición química de Oithona ovalis (calanoideo) alimentado con microalgas (Rosas et al. 1997), el efecto de dietas a base de las microalgas Dunaliella salina, Tetraselmis chuii, Nannochloris oculata y Chorella sp. sobre el crecimiento poblacional del copépodo O. ovalis (Rosas et al. 1998), el efecto de dietas sobre la base de las microalgas $T$. chuii, Isochrysis galbana, Chaetoceros gracilis y N. oculata sobre la fecundidad y longevidad del copépodo ciclopoideo Apocylops distans (Rodríguez 1999).

En este contexto el objetivo de este trabajo fue estudiar el efecto de las microalgas Tetraselmis chuii, Nannochloris oculata y Dunaliella salina sobre el crecimiento poblacional del copépodo Apocyclops distans en diferentes condiciones de temperatura e iluminación.

\section{Materiales y Métodos}

Este trabajo se realizó en la sala de cultivo de peces y crustáceos del Instituto de Investigaciones Científicas de la Universidad de Oriente (IIC-UDO), donde desde hace varios años se realizan experiencias con diferentes grupos del zooplancton, con la finalidad de ser utilizadas como alimento vivo. Esta investigación propone trabajar con una cepa de copépodos aislada de la laguna de Pampatar (Rosas et al. 1993), determinada por la Dra. Evelyn Zoppi (Universidad Central de Venezuela) como, Apocyclops (=Metacyclops) distans. Esta especie se mantuvo cultivada en agua de mar a 40 PSU filtrada por filtros de $0,5 \mu \mathrm{m}$ y esterilizada mediante luz UV $\left(1,5 \mathrm{~L}\right.$. $\left.\mathrm{min}^{-1}\right)$ A partir de allí se tomaron los inóculos $(1 \mathrm{ind} / \mathrm{ml})$ que fueron ubicados en 540 tubos de ensayo de $10 \mathrm{ml}$ de capacidad, conteniendo $5 \mathrm{ml}$ de agua de mar esterilizada en autoclave a $15 \mathrm{psi}, 121{ }^{\circ} \mathrm{C}$, salinidad (40 PSU), inoculada con $3 \times 10^{5} \mathrm{cel} / \mathrm{mL}$ de la cepa de microalga correspondiente a la dieta a ensayar. Las microalgas Tetraselmis chuii, Nannochloris oculata y Dunaliella salina se suministraron desde cultivos unialgales en fase exponencial (4-5 días) mantenidas bajo luz continua provenientes de lámparas fluorescentes a 2500 a 3000 lux y a temperatura de $22 \pm 1^{\circ} \mathrm{C}$, en medio de cultivo preparado con agua de mar natural añadiendo $0,5 \mathrm{~mL} / \mathrm{L}$ de BASF Nitrofoska Foliar ${ }^{\circledR}$, un abono foliar líquido (10-4-7-0,2).

Los materiales de vidrio utilizados se trataron según la metodología descrita por Alfonso \& Leal (1998), se prepararon 540 tubos de ensayo en gradillas, (180 para cada dieta unialgal, tres réplicas por dieta), posteriormente se seleccionaron al azar dos grupos de 270 tubos (90 por cada dieta) para mantenerlos a temperatura ambiente $\left(26-33,8^{\circ} \mathrm{C}\right)$ y la misma cantidad en el laboratorio $\left(22 \pm 1^{\circ} \mathrm{C}\right)$; de cada grupo de 270 se repartieron en subgrupos de 30 tubos por dieta para colocarlos bajo las diferentes condiciones de iluminación (90 tubos bajo luz continua, 90 bajo fotoperíodo y 90 bajo oscuridad), el fotoperíodo fue de 12 h luz y 12 h oscuridad en el laboratorio y se realizó en forma manual tapando las gradillas con una tela de polipropileno color negro. Se estableció 10 días como tiempo experimental basado en la experiencia de Rodríguez (1999) quien determinó que el copépodo $A$. distans completa su ciclo vital en ese tiempo.

El primer muestreo se realizó a las 48 horas para permitir la aclimatación de los copépodos a las nuevas condiciones de cultivo. Una vez iniciados los muestreos, se retiraron 3 tubos de ensayo diariamente por cada tratamiento fijándose su contenido con una mezcla de lugol y formalina al $5 \%$ en la proporción 1:1, la densidad microalgal en los cultivos se determinó con la ayuda de un hemocitómetro (0,1 $\mathrm{mm}$ de profundidad) del tipo Neubauer, marca Boeco, de línea brillante, siguiendo la metodología descrita por Alfonso \& Leal (1998), utilizándose un microscopio binocular Olympus y para el contaje y observación de los copépodos se utilizó una cámara Bogorov con la ayuda de un microscopio estereoscópico Wild-3.

En cada muestra analizada se anotó la densidad celular del alimento para el momento del muestreo, el 
número de nauplios, el número de copepoditos, el número de machos adultos, el número de hembras adultas no ovadas, el número de hembras ovadas, el número de masas ovígeras desprendidas, el número total de hembras y el número total de copépodos. Los datos obtenidos una vez analizados los supuestos de la varianza, fueron transformados utilizándose la raíz cuadrada de $X+0,5$ para su normalización (Zar 1984). Finalmente se realizaron los análisis de varianza multifactorial, así como la prueba a posteriori (LSD).

\section{Resultados y Discusión}

En las tablas 1 y 2 se presentan los máximos valores promedios obtenidos durante este estudio (tercer y sexto día) y la figura 1 que muestra los máximos valores promedios diarios para el número total de copépodos con la especie $T$. chuii.

\section{Alimentación:}

De Mott \& Watson (1991) señalan que los copépodos ciclopoides tienen tasas de alimentación mucho más altas con microalgas móviles que con las no móviles. Abdullahi (1992) señala que en la captura de un gran número de organismos de pequeño tamaño y rápido movimiento es posiblemente un gasto mayor de energía que la usada en la captura de organismos de peso o volumen similares a la del predador; lo que en este estudio podría indicar un menor gasto energético para los copépodos cuando se alimentan con Tetraselmis chuii que fue la microalga utilizada de mayor tamaño y volumen (9 a $10 \mu \mathrm{m}$ de ancho y 12 a $14 \mu \mathrm{m}$ de largo, con 4 flagelos y un movimiento rápido) que para los alimentados con Nannochloris oculata de 1,5 a 2,5 $\mu \mathrm{m}$ de diámetro, esférica, no móvil y Dunaliella salina de 5 a $8 \mu \mathrm{m}$ de ancho y 7 a $12 \mu \mathrm{m}$ de largo, células elongadas con dos flagelos que producen un movimiento lento. Paffenhöfer (1994) demostró que los fitoflagelados representan un recurso alimenticio adecuado para mantener el desarrollo de los estadios juveniles y la producción de huevos de copépodos de la familia cyclopidae.

Santer \& Bosch (1994) indican que las microalgas cocales y las diatomeas fueron menos digeridas que las flageladas al suministrárseles al copépodo ciclopoide Cyclops vicinus. Rodríguez (1999) reportó que Apocyclops distans al igual que muchos ciclopoides se desarrolla bien al ser alimentado con microalgas, teniendo preferencia por las flageladas $T$. chuii e Isochrysis galbana y observó una tendencia hacia la mayor producción de nauplios con las dietas de microalgas flageladas, lo cual es similar a la presente investigación.

\section{Número de nauplios:}

El mayor valor promedio del número de nauplios en este trabajo fue de $105,33 \pm 48,58 / 5 \mathrm{~mL}$ en oscuridad, a temperatura ambiente y cuando fueron alimentados con T. chuii durante el tercer día, el cual estadísticamente resultó ser el día más afectado por las interacciones de los factores de crecimiento especie específicos luz y temperatura, siendo el efecto de la temperatura significativamente importante en el número de nauplios al tercer día $(\mathrm{P} \leq 0,05)$ a valores de 26 a $33,8{ }^{\circ} \mathrm{C}$.

McLaren (1983), Ban (1994) y Santer \& Bosch (1994) indican que los copépodos ciclopoides, calanoides y quetognatos en el plancton marino son poiquilotermos y su desarrollo depende estrictamente de la temperatura, cuando están en presencia de cantidades de alimento igual o en exceso a sus necesidades, afectando también el ciclo de vida de los copépodos Maier (1994), Amarasinghe et al. (1997) señala que la temperatura fue significativa para el crecimiento y desarrollo del copépodo calanoideo Heliodiaptomus viduus cultivado a dos concentraciones de alimento y tres temperaturas $\left(22,5 ; 27,5\right.$ y $\left.32,5{ }^{\circ} \mathrm{C}\right)$.

En el presente trabajo la concentración de alimento ( $\mathrm{P} \leq 0,01)$ utilizada no produjo diferencias sobre el número de nauplios obtenidos, sino que fue afectado favorablemente solo por la temperatura del ambiente. Abdullahi (1992) señala que el tiempo de desarrollo, crecimiento y tamaño de tres especies de copépodos ciclopoides están principalmente controlados por la temperatura.

La luz no produjo diferencias estadística, el máximo promedio del número de nauplios fue en la oscuridad cuando se les suministró $T$. chuii como alimento, estos resultados de cierta manera coinciden con los reportados por Zagorodnyaya \& Kovalev (1988) quien obtuvo la máxima producción de nauplios en oscuridad.

La producción de nauplios de copépodos es muy importante ya que constituyen el principal alimento para la mayoría de las larvas de peces examinadas y en Taiwán la larvicultura ha tenido mucho éxito considerándose que de alrededor de 100 especies que están bajo régimen comercial, el 90 \% están siendo propagadas artificialmente y alimentadas con rotíferos y copépodos entre los que se menciona a Apocyclops 
royi como la especie comúnmente utilizada (Su et al. 1997).

\section{Número de copepoditos:}

La mayor producción de copepoditos fue de 25,33 \pm 11,93 /5mL el sexto día, bajo fotoperíodo a temperatura ambiente, cuando se alimentaron con $T$. chuii. Observándose la preferencia de los copepoditos por las microalgas flageladas de mayor volumen. El análisis estadístico utilizado para los tres factores y sus interacciones mostró que el factor luz, especies y la interacción de ambos produjeron diferencias significativas sobre el número de copepoditos; sin embargo la prueba a posteriori reveló que solo el efecto luz (fotoperíodo) produjo diferencias ( $\mathrm{P} \leq 0,01)$. Su et al. (1997), reportaron que los máximos de mudas en copepoditos de Acartia clausi, ocurrieron en el período comprendido entre 6:00 p.m. y medianoche; este mismo ritmo fue encontrado en copepoditos de Calanus aquae-dulcis. Estas observaciones, junto con los datos publicados sobre copépodos y otros organismos acuáticos y terrestres sugieren que existe un ritmo de reproducción, posiblemente en todos los copépodos y otros animales planctónicos. Aunque como indican Santer \& Bosch (1994) una diferencia entre los nauplios y los copepoditos en cuanto a su sobrevivencia y desarrollo es que los últimos pueden hacerlo a más bajas concentraciones de alimento, además de que responden de manera menos sensitiva que los nauplios a esas bajas densidades de alimento, que en este estudio fue de 1,3 $\mathrm{cel} / \mathrm{mL} \times 10^{5}$ para el sexto día.

Tabla 1

Valores promedio y desviación estándar del número de nauplios (NA/5mL), copepoditos (CO/5mL); machos adultos (MA/5mL); hembras adultas no ovadas (HA/5mL) y hembras ovadas (HO/5mL) de Apocyclops distans alimentados con Tetraselmis chuii, Nannochloris oculata y Dunaliella salina a dos temperaturas y tres condiciones de luz, para el tercer día de experiencia.

Average value and standard deviation of nauplii number (NA/5mL), copepodits (CO/5mL), male adult $(\mathrm{MA} / 5 \mathrm{~mL})$, female no carring eggs (HA/5mL), female carring eggs (HO/5mL), of Apocyclops distans fed on Tetraselmis chuii, Nannochloris oculata and Dunaliella salina at two temperature and three light conditions at third experience day.

\begin{tabular}{|c|c|c|c|c|c|c|c|c|c|c|c|c|}
\hline \multirow[b]{2}{*}{ Especie } & \multirow[b]{2}{*}{ Temperatura } & \multirow[b]{2}{*}{ Luz } & \multicolumn{2}{|c|}{$\mathbf{N}^{0} \mathrm{NA}$} & \multicolumn{2}{|c|}{$\mathrm{N}^{\circ} \mathrm{CO}$} & \multicolumn{2}{|c|}{$\mathbf{N}^{\circ} \mathbf{M A}$} & \multicolumn{2}{|c|}{$\mathbf{N}^{\circ} \mathbf{H A}$} & \multicolumn{2}{|c|}{$\mathrm{N}^{\circ} \mathrm{HO}$} \\
\hline & & & Prom. & Desv & Prom. & Desv. & Prom. & Desv & Prom. & Desv & Prom. & Desv \\
\hline & & F & 3,66 & 5,50 & 2,00 & 2,00 & 1,33 & 1,15 & 9,33 & 3,21 & 9,66 & 5,50 \\
\hline & Ambiente & $\mathrm{O}$ & 105,33 & 48,58 & 1,33 & 1,15 & 8,66 & 2,51 & 12,33 & 15,30 & 5,00 & 1,73 \\
\hline & $26-33,8^{\circ} \mathrm{C}$. & C & 0,00 & 0,00 & 0,00 & 0,00 & 1,66 & 0,57 & 9,00 & 6,00 & 1,33 & 2,30 \\
\hline \multirow[t]{6}{*}{ T. chuii } & & F & 0,00 & 0,00 & 1,33 & 1,52 & 1,00 & 0,00 & 3,66 & 3,21 & 0,00 & 0,00 \\
\hline & Laboratorio & $\mathrm{O}$ & 0,00 & 0,00 & 0,00 & 0,00 & 0,00 & 0,00 & 1,00 & 1,00 & 0,00 & 0,00 \\
\hline & $22 \pm 1^{\circ} \mathrm{C}$ & C & 0,33 & 0,57 & 0,33 & 0,57 & 0,33 & 0,57 & 3,33 & 1,52 & 0,00 & 0,00 \\
\hline & & F & 1,66 & 0,57 & 0,00 & 0,00 & 1,66 & 1,15 & 1,33 & 2,30 & 0,00 & 0,00 \\
\hline & Ambiente & $\mathrm{O}$ & 0,66 & 0,57 & 0,66 & 0,57 & 0,33 & 0,57 & 1,00 & 0,00 & 0,00 & 0,00 \\
\hline & $26-33,8^{\circ} \mathrm{C}$. & C & 4,00 & 3,60 & 0,33 & 0,57 & 0,33 & 0,57 & 0,33 & 0,57 & 0,00 & 0,00 \\
\hline \multirow[t]{6}{*}{ N. oculata } & & F & 2,66 & 3,78 & 3,33 & 0,57 & 0,00 & 0,00 & 0,00 & 0,00 & 0,00 & 0,00 \\
\hline & Laboratorio & $\mathrm{O}$ & 0,00 & 0,00 & 0,00 & 0,00 & 0,33 & 0,57 & 0,33 & 0,57 & 0,00 & 0,00 \\
\hline & $22 \pm 1^{\circ} \mathrm{C}$ & $\mathrm{C}$ & 2,33 & 2,30 & 1,00 & 1,73 & 0,66 & 0,57 & 0,66 & 1,15 & 0,00 & 0,00 \\
\hline & & F & 30,33 & 33,94 & 0,00 & 0,00 & 1,33 & 0,57 & 2,33 & 2,08 & 1,00 & 1,73 \\
\hline & Ambiente & $\mathrm{O}$ & 7,00 & 12,12 & 0,00 & 0,00 & 2,33 & 0,57 & 0,66 & 0,57 & 0,00 & 0,00 \\
\hline & $26-33,8^{\circ} \mathrm{C}$. & $\mathrm{C}$ & 4,66 & 5,68 & 0,33 & 0,57 & 1,66 & 1,15 & 0,33 & 0,57 & 0,33 & 0,57 \\
\hline \multirow[t]{3}{*}{ D. salina } & & F & 7,66 & 13,27 & 0,33 & 0,57 & 0,66 & 1,15 & 0,33 & 0,57 & 0,00 & 0,00 \\
\hline & Laboratorio & $\mathrm{O}$ & 0,00 & 0,00 & 0,66 & 1,15 & 2,00 & 1,00 & 0,66 & 1,15 & 0,00 & 0,00 \\
\hline & $22 \pm 1^{\circ} \mathrm{C}$ & C & 2,66 & 3,78 & 0,66 & 1,15 & 1,00 & 1,00 & 1,33 & 0,57 & 0,00 & 0,00 \\
\hline
\end{tabular}

F: fotoperíodo O: oscuridad C: luz continua. 
Tabla 2

Valores promedio y desviación estándar del número de nauplios (NA/5mL), copepoditos (CO/5mL); machos adultos (MA/5mL); hembras adultas (HA/5mL) y hembras ovadas (HO/5mL) de Apocyclops distans alimentados con Tetraselmis chuii, Nannochloris oculata y Dunaliella salina a dos temperaturas y tres condiciones de luz, para el sexto día de experiencia.

Average value and standard desviation of nauplii number $(\mathrm{NA} / 5 \mathrm{ml})$, copepodits $(\mathrm{CO} / 5 \mathrm{~mL})$, male adult $(\mathrm{MA} / 5 \mathrm{~mL})$, female no carring eggs $(\mathrm{HA} / 5 \mathrm{~mL})$, female carring eggs $(\mathrm{HO} / 5 \mathrm{~mL})$, of Apocyclops distans fed on Tetraselmis chuii, Nannochloris oculata and Dunaliella salina at two temperature and three light conditions at sixth experience day.

\begin{tabular}{|c|c|c|c|c|c|c|c|c|c|c|c|c|}
\hline & \multirow{2}{*}{\multicolumn{2}{|c|}{$\mathbf{N}^{\circ} \mathbf{N A}$}} & \multirow{2}{*}{\multicolumn{2}{|c|}{$\mathrm{N}^{\circ} \mathrm{CO}$}} & \multirow{2}{*}{\multicolumn{2}{|c|}{$\mathbf{N}^{0}$ MA }} & \multirow{2}{*}{\multicolumn{2}{|c|}{$\mathbf{N}^{\circ} \mathbf{H A}$}} & \multirow{2}{*}{\multicolumn{2}{|c|}{$\mathrm{N}^{\circ} \mathrm{HO}$}} \\
\hline & & & & & & & & & & & & \\
\hline \multirow[t]{4}{*}{ Especie } & Temperatura & Luz & Prom. & Desv & Prom. & Desv. & Prom. & Desv & Prom. & Desv & Prom. & Desv \\
\hline & & F & 51,00 & 48,28 & 25,33 & 11,93 & 12,33 & 8,14 & 26,66 & 36,66 & 0,66 & 0,57 \\
\hline & Ambiente & $\mathrm{O}$ & 33,33 & 29,02 & 0,00 & 0,00 & 9,00 & 6,24 & 18,00 & 17,43 & 0,00 & 0,00 \\
\hline & $26-33,8^{\circ} \mathrm{C}$. & $\mathrm{C}$ & 8,33 & 10,40 & 2,00 & 3,46 & 4,00 & 3,60 & 11,66 & 14,22 & 1,66 & 1,52 \\
\hline \multirow[t]{6}{*}{ T. chuii } & & $\mathrm{F}$ & 7,33 & 9,45 & 8,66 & 8,08 & 2,00 & 1,00 & 1,33 & 0,57 & 1,00 & 1,00 \\
\hline & Laboratorio & $\mathrm{O}$ & 1,66 & 2,88 & 0,00 & 0,00 & 1,66 & 2,88 & 1,66 & 1,15 & 0,33 & 0,57 \\
\hline & $22 \pm 1^{\circ} \mathrm{C}$ & $\mathrm{C}$ & 0,33 & 0,57 & 0,00 & 0,00 & 4,33 & 3,21 & 2,33 & 1,52 & 1,00 & 1,00 \\
\hline & & $\mathrm{F}$ & 30,66 & 10,59 & 1,33 & 1,52 & 2,66 & 2,08 & 5,33 & 6,80 & 3,00 & 1,00 \\
\hline & Ambiente & $\mathrm{O}$ & 0,33 & 0,57 & 1,00 & 1,73 & 0,66 & 1,15 & 1,00 & 1,73 & 0,00 & 0,00 \\
\hline & $26-33,8^{\circ} \mathrm{C}$. & $\mathrm{C}$ & 7,66 & 8,02 & 0,66 & 1,15 & 1,33 & 0,57 & 0,66 & 0,57 & 0,33 & 0,57 \\
\hline \multirow[t]{6}{*}{ N. oculata } & & $\mathrm{F}$ & 0,33 & 0,57 & 3,33 & 3,51 & 0,33 & 0,57 & 0,00 & 0,00 & 0,00 & 0,00 \\
\hline & Laboratorio & $\mathrm{O}$ & 0,33 & 0,57 & 0,33 & 0,57 & 0,00 & 0,00 & 0,00 & 0,00 & 0,00 & 0,00 \\
\hline & $22 \pm 1^{\circ} \mathrm{C}$ & $\mathrm{C}$ & 1,00 & 1,00 & 1,33 & 1,15 & 1,33 & 0,57 & 0,33 & 0,57 & 0,00 & 0,00 \\
\hline & & $\mathrm{F}$ & 0,00 & 0,00 & 2,33 & 3,21 & 1,66 & 1,52 & 0,33 & 0,57 & 1,00 & 0,00 \\
\hline & Ambiente & $\mathrm{O}$ & 6,66 & 9,81 & 0,33 & 0,57 & 2,33 & 2,51 & 2,00 & 1,00 & 0,00 & 0,00 \\
\hline & $26-33,8^{\circ} \mathrm{C}$. & $\mathrm{C}$ & 16,66 & 18,14 & 0,33 & 0,57 & 1,33 & 0,57 & 1,33 & 0,57 & 0,00 & 0,00 \\
\hline \multirow[t]{3}{*}{ D. salina } & & F & 11,33 & 8,38 & 0,66 & 0,57 & 2,33 & 0,57 & 1,66 & 1,52 & 0,00 & 0,00 \\
\hline & Laboratorio & $\mathrm{O}$ & 5,66 & 4,93 & 0,33 & 0,57 & 2,33 & 1,52 & 2,00 & 2,00 & 0,00 & 0,00 \\
\hline & $22 \pm 1^{\circ} \mathrm{C}$ & $\mathrm{C}$ & 17,33 & 10,06 & 1,00 & 1,73 & 0,66 & 0,57 & 1,15 & 1,00 & 0,33 & 0,57 \\
\hline
\end{tabular}

F: fotoperíodo O: oscuridad C: luz continua. 


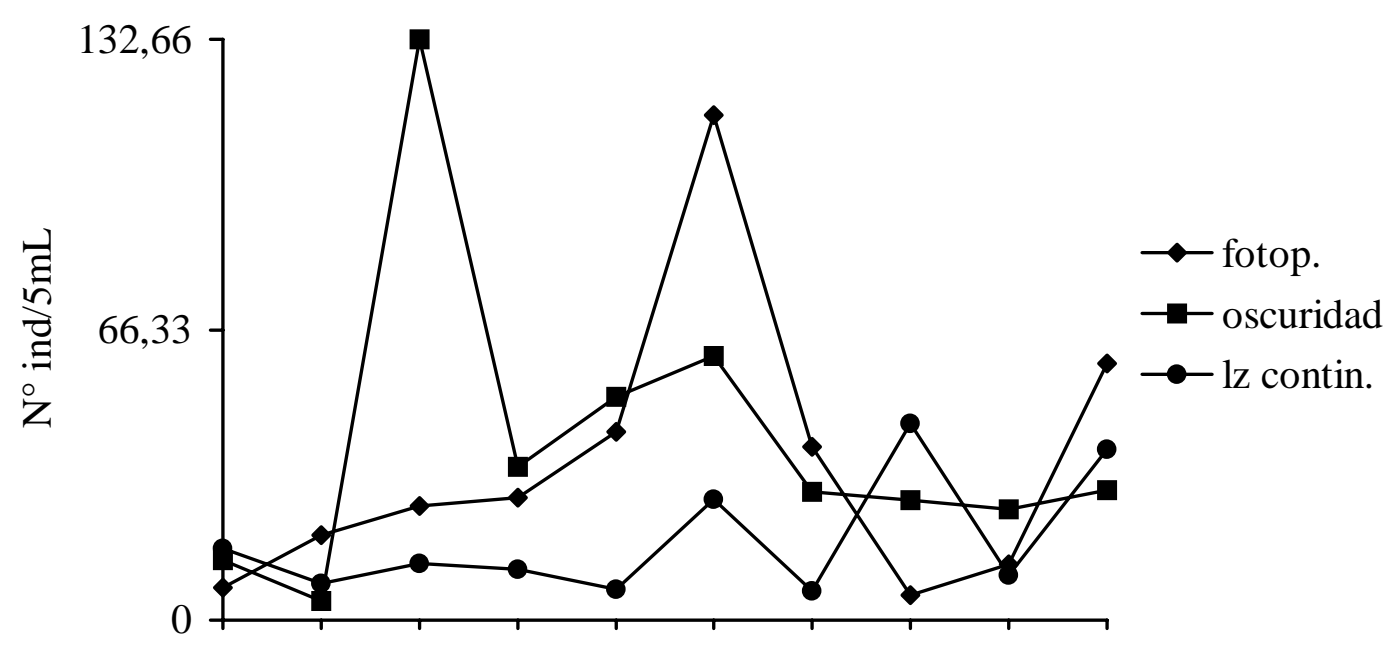

a

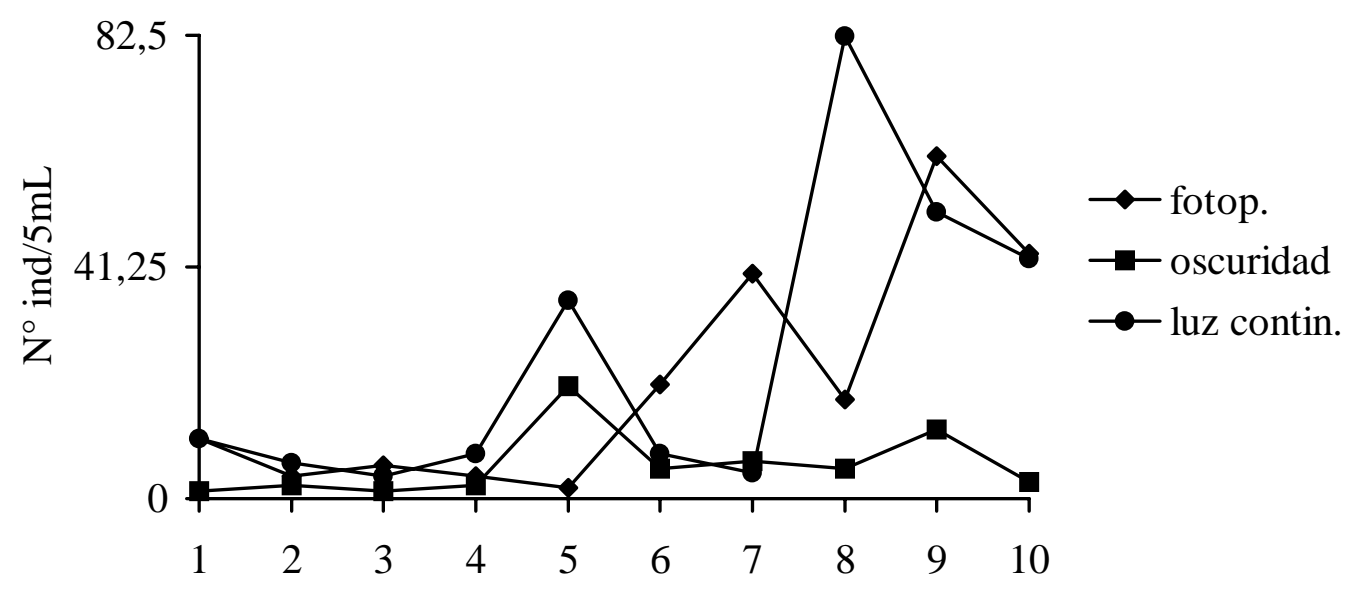

Días

Figura 1

Valores promedios del número total de individuos $\left(\mathrm{N}^{\circ}\right.$ ind/5mL) de Apocyclops distans alimentados con $T$. chuii a dos temperaturas (a: ambiente; b: laboratorio) y tres condiciones de luz (fotoperíodo, oscuridad y luz continua).

Average value of total individuals number (Ind./5mL) of Apocycloops distans fed with T. chuii at two different temperatures (a: natural condition, b: laboratory condition) and three light conditions (photoperiod, dark and continous light)

Para determinar el efecto de las especies formó dos grupos homogéneos $(\mathrm{P} \leq 0,05)$, uno con $T$. chuii y $N$. oculata y el otro grupo con $N$. oculata y D. salina; aunque $T$. chuii y $N$. oculata no produjeron diferencias estadística entre ellas, los mayores valores promedio para ese día se produjeron con T. chuii. Estos resultados podrían explicarse según Abdullahi (1992) quien señala que existe un menor gasto energético en la captura de microalgas de peso y volumen similares al predador.

\section{Copépodos machos adultos:}

El valor promedio máximo para los machos adultos fue de $12,33 \pm 8,14 \mathrm{MA} / 5 \mathrm{~mL}$ y se produjo al sexto día de cultivo, bajo fotoperíodo a temperatura ambiente cuando se alimentaron con T. Chuii. Los efectos especies y temperatura fueron los que produjeron diferencias ( $\mathrm{P} \leq$ $0,01)$; formando para el efecto especies, dos grupos homogéneos, uno con la microalga $T$. chuii que produjo 
los mayores valores promedios y el otro menor por $N$. oculata y D. salina. Respecto a la temperatura se formaron dos grupos homogéneos produciéndose los mayores valores promedio a temperatura ambiente. Abdullahi (1992) asegura que el tiempo de desarrollo, crecimiento y tamaño de los adultos de tres especies de copépodos son principalmente controlados por la temperatura.

\section{Copépodos hembras adultas no ovadas:}

El mayor número de hembras adultas no ovadas (26,66 $\pm 36,66 / 5 \mathrm{~mL}$ ) se produjo el sexto día superando a los machos adultos en una proporción de 2:1, los efectos especies y temperatura fueron los que influyeron el número de hembras adultas no ovadas, la prueba $a$ posteriori $(\mathrm{P} \leq 0,01)$ formó entre las especies dos grupos homogéneos, uno con $T$. chuii con los mayores valores promedio y el otro grupo formado con $N$. oculata y $D$. salina que produjeron valores promedio más bajos; para el efecto temperatura existen dos grupos homogéneos siendo los valores promedios más altos a temperatura ambiente $(\mathrm{P} \leq 0,01)$.

Moore (1983), Kiørboe \& Sabatini (1994) y Sabatini \& Kiørboe (1994) indican que la relación desigual de sexos en los copépodos marinos en cultivos de laboratorio puede ser 2:1 pero que en poblaciones naturales puede ser 6:1 y que los machos pueden morir inmediatamente después de la cópula. Davis (1986) señala que en la mayoría de las especies de copépodos el número de hembras excede al número de machos debido a que estos últimos tienen un tiempo de vida más corto. Mullin \& Brooks (1967) concluyen que los machos viven por períodos de vida más cortos que las hembras, aun en presencia de alimento en exceso y Rodríguez (1999) indicó que Apocyclops distans no mostró una proporción sesgada hacia ninguno de los dos sexos, sin embargo en el presente estudio se observó que el número de machos adultos fue menor comparado con el número de hembras en todas las dietas suministradas y bajo todas las condiciones de cultivo ensayadas.

\section{Copépodos hembras adultas ovadas:}

El mayor número promedio de hembras ovadas se observó el tercer día bajo fotoperíodo a temperatura ambiente $(9,66 \pm 5,50 / 5 \mathrm{~mL})$ cuando fueron alimentados con Tetraselmis chuii. Los efectos especies y temperatura resultaron ser los que afectaron el número de hembras ovadas; formando dos grupos homogéneos ( $\mathrm{P} \leq 0,01)$ uno con $T$. chuii que produjo los mayores valores promedio y el otro con $N$. oculata y D. salina, con los menores valores promedio; para la temperatura se formaron dos grupos homogéneos produciéndose los mayores valores promedio en el ambiente.
Posiblemente los materiales energéticos suministrados por la microalga $T$. chuii solo fue suficiente para que un promedio de 9,66 hembras produjeran masas ovígeras, lo que explicaría el mayor número promedio de hembras adultas no ovadas $\mathrm{y}$ machos adultos, los cuales no requieren un gasto extra de energía debido a que no realizan la formación de oviductos en el caso de los machos o la producción de huevos en el caso de las hembras (Jamieson 1980).

Rodríguez (1999) encontró que estadísticamente no existe diferencias entre el número de huevos portados por hembra y el alimento suministrado, sin embargo obtuvo los mayores valores con las microalgas flageladas. En el presente trabajo aunque no se determinó el número de huevos portados por hembras, si se contabilizó el número de masas ovígeras sueltas, encontrándose que el mayor valor promedio (27,33 \pm $17,92 / 5 \mathrm{~mL}$ ) se obtuvo junto con la mayor concentración de $N$. oculata $\left(16,93 \mathrm{cel} / \mathrm{mL} \times 10^{5}\right)$, el segundo día de experiencia, bajo fotoperíodo a temperatura ambiente. Al respecto Sabatini \& Kiørboe (1994) señalan que para el copépodo ciclopoide Oithona similis el número total de masas ovígeras y de huevos liberados en toda su historia de vida (desde su captura) y el de todo el período reproductivo aumenta con el aumento de la concentración de alimento; también indicó que la fecundidad y el tiempo de vida de las hembras son influenciados por la concentración del alimento indicando que en su estudio un 80 \% de las hembras, fue verdaderamente reproductivas al más alto nivel de alimento ensayado.

Sabatini \& Kiørboe (1994) resaltan que los copépodos alimentados con el dinoflagelado heterotrófico Oxyrrhis marina como suplemento de la dieta suministrada produjeron un nuevo saco o masa ovígera inmediatamente después de liberar el anterior. Maier (1995) indicó que las hembras de Cyclops vicinus producen masas ovígeras de aproximadamente 60 huevos cada segundo día en intervalos de tres y Metacyclops minutus produce masas ovígeras de 30 huevos cada primer día en intervalos de dos días.

\section{Total de copépodos:}

La mayor producción del total de hembras (19,00 \pm 4,00 $/ 5 \mathrm{~mL}$ ) ocurrió el sexto día a temperatura ambiente bajo fotoperíodo al ser alimentadas con $T$. chuii, con diferencias significativas para los efectos especies $y$ temperatura, la especie $T$. chuii a temperatura ambiente produjo los mayores valores promedio ( $\mathrm{P} \leq 0,01)$. En los copépodos ciclopoides como se señalo anteriormente por lo general se produce un sesgo hacia el mayor número de hembras adultas que de machos (Kiørboe \& Sabatini 1994), resultados estos que coinciden con los obtenidos en esta investigación. 
El número total de copépodos a temperatura ambiente fue mayor en la oscuridad al tercer día (132,66 $\pm 34,00 \mathrm{ind} / 5 \mathrm{~mL}$ ) cuando fueron alimentadas con $T$. chuii (fig. 1), la interacción entre los efectos luz, especies de microalgas y temperatura produjo diferencias $(\mathrm{P} \leq 0,01)$ y se debieron principalmente al factor luz y las especies de microalgas utilizadas.

\section{Conclusiones}

Según los resultados obtenidos en este estudio el copépodo Apocyclops distans pudo desarrollarse completamente y presentó una evidente preferencia por la especie Tetraselmis chuii (Fig. 1), microalga de mayor volumen celular y movilidad entre las tres utilizadas. Las microalgas $N$. oculata y $D$. salina produjeron valores promedio bajos en el número total de copépodos, aunque permitieron el desarrollo completo de A. distans.

La temperatura fue el factor que produjo diferencias significativas ( $\mathrm{P} \leq 0,01)$ sobre el número de nauplios, con un máximo valor promedio de 105,33 \pm 48,58/5mL al tercer día a temperatura ambiente cuando se alimentaron con $T$. chuii (Tabla 1 ).

El factor luz presentó diferencias significativas ( $\mathrm{P} \leq$ 0,01 ) sobre el número de copepoditos obtenidos, siendo $25,33 \pm 11,93 / 5 \mathrm{~mL}$ el máximo valor promedio bajo fotoperíodo el sexto día (Tabla 2).

El mayor número de machos adultos y hembras adultas no ovadas se obtuvo al sexto día cuando fueron alimentados con $T$. chuii a temperatura ambiente y la luz fue un factor que no produjo diferencias significativas (Tabla 2).

El número de hembras adultas no ovadas producidas el sexto día fue de $26,33 \pm 36,66 / 5 \mathrm{~mL}$ y superó al número de machos $(12,33 \pm 11,93 / 5 \mathrm{~mL})$ para ese mismo día en una proporción 2:1 (Tabla 2).

El número máximo de hembras adultas ovadas (9,66 $\pm 3,21 / 5 \mathrm{~mL}$ ) se obtuvo el tercer día al ser alimentadas con $T$. chuii a temperatura ambiente y el mayor promedio del número de masas ovígeras desprendidas fue de 27,33 $\pm 17,92 / 5 \mathrm{~mL}$ a temperatura ambiente alimentado con $N$. oculata (Tabla 1 ).

El número total máximo de copépodos $(132,66 \pm$ $34,00 / 5 \mathrm{~mL}$ ) se produjo el tercer día cuando fueron alimentados con $T$. chuii a temperatura ambiente y en la oscuridad produciéndose diferencias significativas $(\mathrm{P} \leq$ 0,01) debido a la interacción de los tres factores estudiados (Tabla 1).

\section{Literatura Citada}

Abdullahi B. 1992. Effects of diet on growth and development of three species of cyclopoid copepods. Hydrobiologia 232: 233-241.

Alfonso E \& S Leal. 1998. Creación y mantenimiento de un cepario de microalgas, 21 p. Centro de Investigaciones marinas. Universidad de la Habana.

Amarasinghe B, M Boersma \& J Vijverberg. 1997. The Effect of temperature, and food quantity and quality on the growth and development rates in laboratory - cultured copepods and cladoceraus from a Sri Lankan reservoir. Hidrobiología 350: 131-144.

Ban S. 1994. Effect of temperature and food concentration on post-embrionic development, egg production and adult body size of calanoid copepod Eurytemora affinis. Journal of Plankton Research 16 (6): 721-735.

Davis C. 1986. Planktonic copepoda (lncluding Monstrilloida). En: Steidinger K \& L Walker. Marine plankton life cycle strategies: 67-92. CRC Press Inc, Boca Ratón, Florida.

De Mott W \& M Watson. 1991. Remote detection of algae by copepods: responses to algal size, odors and motility. Journal of Plankton Research 13: 1203-1222.

Heath P \& C Moore. 1997. Rearing dover sole larvae on Tisbe and Artemia diets. Aquaculture International 5: 29-39.

Jamieson C. 1980. Observations on the effect of diet and temperature on rate of development of Mesocyclps leuckarti (Claus) (Copepoda, Cyclopoida). Crustaceana 38 (2): 145-154.

Kiefer F. 1956. Freilebende Ruderfusskrebse (Crustacea, Copepoda) I. Calanoida und Cyclopoida. Ergebnisse der Deutschen Limnologischen Venezuela-Expedition, 1952, 1: 233-268.

Kiørboe T \& M Sabatini. 1994. Reproductive and life cycle strategies in egg-carrying cyclopoid and free-spawning calanoid copepods. Journal of Plankton Research 15 (1): 37-55.

Lavens P \& P Sorgeloos. 1996. Manual on the production and use of live food for Aquaculture. FAO: 319-357, Bélgica.

Maier G. 1994. Patterns of life history among cyclopoid copepods of Central Europe. Freshwater Biology 31 (1): 77-86.

Maier G. 1995. Mating frequency and interspecific mating in some freshwater cyclopoid copepods. Oecología 101: 245250.

McLaren I. 1983. Some relationships between temperature and egg size, body size, development rate and fecundity, of the copepod Pseudocalanus. Limnology and Oceanography 10: 528-538.

Moore E. 1983. Physioecology of tropical marine copepods. II Sex ratios. Crustaceana 44 (2): $113-122$. 
Mullin M \& E Brooks. 1967. Laboratory culture, growth rate, and feeding behavior of a planktonic marine copepod. Limnology and Oceanography 12: 657-666.

Paffenhöfer G A. 1994. Variability due to feeding activity of individual copepods. Journal of Plankton Research 16 (6): 617-626.

Rodríguez E. 1999. Efectos de cuatro dietas a base de microalgas sobre algunos aspectos reproductivos, longevidad, longitud corporal y proporción de sexos de Apocyclops distans (Kiefer, 1956) (Copepoda, Cyclopoidae). Trabajo para Licenciado, 70 p. Cumaná, Venezuela.

Rosas J, O Gómez \& A Gómez. 1993. El uso de copépodos en Acuicultura. Acta Científica Venezolana 44: 4.

Rosas J, J Millán \& T Cabrera. 1997. Mix Culture and chemical Composition of The Rotifer Brachionus plicatilis and the Copepodite Oithona ovalis rearing in the microalgae Tetraselmis chuii Island Aquaculture and Tropical Aquaculture: 265-266. Les trois Islets, Martinique.
Rosas J, T Cabrera \& J Millán. 1998. Efecto de cuatro especies de microalgas en el crecimiento poblacional del copépodo Oithona ovalis Herbst 1955 (Crustacea: Copepoda) en el laboratorio. Revista de Biología Marina y Oceonografía 33 (2): 313-323.

Sabatini M \& T Kiørboe. 1994. Egg production, growth and development of the cyclopoid copepod Oithona similis. Journal of Plankton Research 16 (10): 1329-1351.

Santer B \& F Bosch. 1994. Herbivorous nutrition of ciclops vicinus: the effect of pure algal diet on feeding, development, reproduction and life cycle. Journal of Plankton Research 16 (2): 171-195.

Stottrup J \& N Norsker. 1997. Production and use of copepods in fish larviculture. Aquaculture 155: 231-247.

Su H, M Su \& I Liao. 1997. Collection and culture of live foods for aquaculture in Taiwan. Hydrobiologia 358: 3740.

Zagorodnyaya Y \& A Kovalev. 1988. Diurnal rhythm in reproduction and ontogenetic changes in marine copepods. Hydrobiological Journal 24 (2):15-18.

Zar J. 1984. Biostatistical analysis, 718 p. Prentice Hall Englewood Cliffs, Nueva York.

Recibido en septiembre de 2001 y aceptado en noviembre de 2001 